\title{
Phenotypical diversity of airway morphology in chronic lung graft vs. host disease after stem cell transplantation
}

\author{
Stijn E. Verleden $\mathbb{1}^{1} \cdot$ John E. McDonough ${ }^{1} \cdot$ Helene Schoemans ${ }^{2} \cdot$ Christiane Knoop $^{3} \cdot$ Johny Verschakelen $^{4}$. \\ Adriana Dubbeldam ${ }^{4}$ - Matthieu N. Boone ${ }^{5}$. Luc Van Hoorebeke ${ }^{5}$. Erik Verbeken ${ }^{4}$ - Birgit Weynand ${ }^{4}$. \\ Dirk Van Raemdonck $\mathbb{B}^{1} \cdot$ Geert M. Verleden ${ }^{1} \cdot{\text { Robin } \operatorname{Vos}^{1} \cdot \text { Bart M. Vanaudenaerde }}^{1}$
}

Received: 8 October 2018 / Revised: 10 December 2018 / Accepted: 11 December 2018 / Published online: 5 February 2019

(c) United States \& Canadian Academy of Pathology 2019

\begin{abstract}
Pulmonary graft vs. host disease is a diverse and underestimated complication following allogenic hematopoietic stem cell transplantation. We aimed to compare the airway architecture with chronic lung allograft dysfunction post lung transplantation. Inflated explant lungs from graft vs. host disease patients were compared with lungs with chronic lung allograft dysfunction following lung transplantation, and control lungs using a combination of CT, microCT, and histology ( $n=6$ per group) and pathology in the (small) airways was further quantified and analyzed. Following allogenic hematopoietic stem cell transplantation, three patients presented as bronchiolitis obliterans syndrome and three patients showed interstitial changes and restriction. The CT analysis demonstrated a strong similarity between bronchiolitis obliterans syndrome after lung transplantation and post allogenic hematopoietic stem cell transplantation, evidenced by severe $(>50 \%)$ airway obstruction from generation 9 , with $70.8 \%$ of the airways ending in obstruction. Further analysis indicated that the airways either collapsed or accumulated matrix along a segment of the airway. In patients with restriction and interstitial changes following allogenic hematopoietic stem cell transplantation, the degree of airway obstruction was lower compared with bronchiolitis obliterans syndrome post allogenic hematopoietic stem cell transplantation, but similar to restrictive allograft syndrome post lung transplantation, showing a lower proportion of airway obstruction (20-35\%), decreased number of terminal bronchioles per lung $(p<0.01)$, and parenchymal fibrosis. We observed similarities in the airway and parenchymal morphometric changes in lung graft vs. host disease and with chronic lung allograft dysfunction following lung transplantation, suggesting similar pathophysiological mechanisms.
\end{abstract}

Stijn E. Verleden

stijn.verleden@med.kuleuven.be

1 Lung Transplant Unit, Department of Chronic diseases, Metabolism and Aging, KU Leuven, Leuven, Belgium

2 Department of Hematology, University Hospital Leuven and $\mathrm{KU}$ Leuven, Leuven, Belgium

3 Unite de Transplantation Cardiaque et Pulmonaire, Université Libre de Bruxelles, Brussels, Belgium

4 Department of Imaging and Pathology, KU Leuven, Leuven, Belgium

5 Department of Physics and Astronomy, Radiation Physics-Centre for X-ray Tomography, Ghent University, Ghent, Belgium

\section{Introduction}

Allogeneic hematopoietic stem cell transplantation is a curative option for patients suffering from a range of hematologic malignancies. Although long-term survival is significantly improving over the last decades [1,2], graft vs. host disease remains a major concern, considering its significant impact on long-term mortality, morbidity, and quality of life [3, 4]. The incidence of lung graft vs. host disease is rather low with about $2-5 \%$ of allogenic hematopoietic stem cell transplantation recipients being diagnosed, increasing to about $15 \%$ in patients with chronic graft vs. host disease elsewhere [5]. Lung graft vs. host disease is known to have a dismal prognosis [6-8] but it is often difficult to diagnose, as it only becomes symptomatic at an advanced stage and serial pulmonary function testing is not always part of routine clinical follow-up. The NIH 2014 consensus criteria advocated its pre-clinical detection 
based on pulmonary function, open lung biopsy, or airtrapping in combination with graft vs. host disease elsewhere, commonly referred to as bronchiolitis obliterans syndrome [9]. A number of authors, however, have reported a heterogeneous group of diffuse diseases as potential manifestations of graft vs. host disease [10]. The NIH criteria indeed recognized the presence of other atypical presentations such as cryptogenic organizing pneumonia and progressive restrictive lung disease [9], but clear definitions of these entities are still lacking in the post allogenic hematopoietic stem cell transplantation setting. Recent studies have described incidence and prognosis of patients with progressive interstitial changes on computed tomography (CT) after allogenic hematopoietic stem cell transplantation and a restrictive pulmonary function defect, reporting that between 12 and $60 \%$ of patients with lung graft vs. host disease present with interstitial changes on CT [11].

A similar subdivision has recently been proposed for chronic lung allograft dysfunction following lung transplantation [12], one of the main barriers preventing longterm survival [13]. Chronic lung allograft dysfunction is believed to be the clinical correlate of chronic lung rejection. Next to the most prevalent, obstructive phenotype of chronic lung allograft dysfunction (also referred to as bronchiolitis obliterans syndrome), a restrictive allograft syndrome has been proposed as a separate phenotype, characterized by a restrictive pulmonary function defect, persistent interstitial abnormalities, and a poor survival [14].

At the microscopic level, obliterative bronchiolitis is the typical pathological hallmark in lungs from patients with pulmonary graft vs. host disease and the different phenotypes of chronic lung allograft dysfunction [15-17]. Obliterative bronchiolitis is characterized by pathological scarring of the (small) airway, initiated by persistent injury to the airway epithelium, leading to an excessive immune response and ultimately complete obliteration of the airway [18]. Given that obliterative bronchiolitis explains the poor pulmonary function and increased airway resistance, we investigated the site and nature of airway disease in explant bronchiolitis obliterans syndrome and restrictive allograft syndrome lungs at redo-transplantation, where we previously demonstrated that in bronchiolitis obliterans syndrome about $50-60 \%$ of the airways are obstructed from generation 7 on [19]. In restrictive allograft syndrome, a lower degree of obstruction was found, but normal airways were reduced in areas of fibrosis and a lower number of terminal bronchioles was found [20].

We therefore decided to conduct a comparative analysis of airway morphometrical changes in obstructive and restrictive phenotypes of lung rejection after allogenic hematopoietic stem cell transplantation and lung transplantation, to better understand the morphometric similarities and differences.

\section{Material and methods}

\section{Patient selection}

Explant lungs were collected from patients undergoing primary or secondary lung transplantation for end-stage rejection following stem cell and lung transplantation at either UZ Gasthuisberg, Leuven, Belgium or Erasme Hospital, Brussels, Belgium. Explant lungs at redo lung transplantation for chronic lung allograft dysfunction were matched to the graft vs. host disease group according to age and gender. Unused donor lungs were also matched and included as controls. In addition, one patient that underwent allogenic hematopoietic stem cell transplantation without graft vs. host disease, but who decided to go for palliative sedation for extrapulmonary complications, was included as control. All patients provided written informed consent and this study was approved by the hospitals ethical committee (S52174). Donor lungs were collected following Belgian legislation where declined donor lungs can be released for research.

Within chronic rejection post lung transplantation, restrictive allograft syndrome was defined as patients with a persistent decline in forced expiratory volume in $1 \mathrm{~s} \geq 20 \%$ of the post-operative best, accompanied by a restrictive pulmonary function defect (total lung capacity decrease $\geq$ $10 \%$ or forced vital capacity decrease $\geq 20 \%$ compared with baseline) and persistent interstitial abnormalities on CT [21]. Bronchiolitis obliterans syndrome was defined as a strictly obstructive pulmonary function without interstitial abnormalities on CT. The assignment of the groups was confirmed by pathological examination of the contralateral lung. Part of this lung collection has been used in previous publications [19, 20], but new cases (bronchiolitis obliterans syndrome, $n=1$; restrictive allograft syndrome, $n=1$; control, $n=4$; graft vs. host disease, $n=6$ ) were included for this analysis to allow for patient-specific matching.

\section{Lung processing}

Lungs were processed as described previously [19, 20]. In brief, lung were air-inflated at $30 \mathrm{~cm} \mathrm{H}_{2} \mathrm{O}$ and after deflation to $10 \mathrm{~cm} \mathrm{H}_{2} \mathrm{O}$, lungs were fixed in the fumes of liquid nitrogen at constant airway pressure and preserved at $-80{ }^{\circ} \mathrm{C}$ and scanned with CT in frozen condition (Siemens Somatom, 1.0 mm slice thickness, B60F window). This CT was used to manually count the number of airways per generation [19, 20]. In addition, lung volume, density, and weight was determined using custom-made software. Pre-operative in vivo CT scans were scored by a blinded radiologist (AD) using the Fleischner Society guidelines [22]. After CT scan, lungs were sliced using a bandsaw from apex to base and were systematically sampled using a core bore or power drill. To enable the comparison of anatomical similar locations, we used a random 
number generator to select cores for this study. Two cores were selected from the upper zones (slice 3 and 4) and two cores from the lower lung zones (slice 7-8). These cores were scanned in frozen condition $\left(-30^{\circ} \mathrm{C}\right)$ using microCT (Skyscan 1172, Bruker, Kontich, Belgium). The microCT scan was further used to manually count the number of terminal bronchioles (defined anatomically as the last conducting airway) and to measure surface density, tissue volume, and tissue percentage using CTan (Bruker, Kontich, Belgium).

To improve the spatial resolution, from two additional explants from patients with lung graft vs. host disease (one obstructive graft vs. host disease, one restrictive graft vs. host disease), the contralateral (right) explant lung was separated in the upper/middle and lower lobe. Both lobes were separately air-inflated and air-dried for 1 week using constant air pressure. The dried specimens were then scanned using the in-house developed microCT system HECTOR [23] operated at $120 \mu \mathrm{m}$ voxel size, tube voltage $80 \mathrm{kV}, 208 \mu \mathrm{A}$, and reconstructed using Octopus Reconstruction (XRE, Gent, Belgium). These images were loaded into ITK-SNAP for semi-automated segmentation of the airways, where obstructions were indicated manually. NEURONstudio was used to assess the number of terminal airways.

\section{Histology processing}

To obtain histology of the specimens that underwent microCT, the tissue core was fixed in $4 \%$ paraformaldehyde in $96 \%$ acetone, dehydrated in increasing ethanol gradients, and embedded in paraffin. Slicing was performed using a microtome $(8 \mu \mathrm{m})$ and subsequently stained with hematoxylin and eosin (H\&E).

\section{Statistical analysis}

Results are expressed as median and interquartile range or mean \pm SD. The number of terminal bronchioles, the surface density, and $\%$ of tissue were averaged over the entire lung. Differences between bronchiolitis obliterans syndrome, restrictive allograft syndrome, and controls were tested with Kruskall-Wallis analysis of variance (ANOVA) and Dunn's post hoc test. The number of airways per generation was compared using two-way ANOVA with Tukey's post hoc test (Graphpad Prism 4.0, Graphpad, CA, USA). A $p$-value $<0.05$ was considered significant.

\section{Results}

\section{Patient characteristics}

Six lungs were included in each group and were well matched for baseline characteristics including age, gender, and height
(Table 1). Median time between allogenic hematopoietic stem cell transplantation and lung transplantation was $4.8 \mathrm{Y}$ (3.3-10.3). The allogenic hematopoietic stem cell transplantation group was quite heterogeneous with three patients showing a severe obstructive pulmonary function defect without severe consolidation on CT (referred to as obstructive graft vs. host disease) and three patients showing a more restrictive pulmonary function defect with lung deformation ground glass opacification and consolidation on CT (restrictive graft vs. host disease) (Table 2, Fig. 1). Five patients also had severe extrapulmonary graft vs. host disease manifestation with skin manifestations $(n=2)$, kidney $(n=1)$, gastrointestinal system $(n=1)$, mouth $(n=1)$, eye $(n=1)$, and joints $(n=1)$. There were no differences in pulmonary function with all patients suffering from end-stage lung disease, except for a lower forced expiratory volume in $1 \mathrm{~s} /$ forced vital capacity in bronchiolitis obliterans syndrome post lung transplantation $(p=0.0008)$, whereas on CT bronchiolitis obliterans syndrome following lung transplantation was also characterized with a lower degree of reticulation $(p=0.013)$ and volume deformation $(p=0.049)$.

\section{CT airway counts}

Manual counting of the airways based on the ex vivo lung CT scan (resolution of $700 \mu \mathrm{m}$ ) indicated significant differences between groups. The graft vs. host disease group showed an increase in the number of airways and restrictive allograft syndrome post lung transplantation showed a decrease in the number of airways $(p<0.0001)$ compared with bronchiolitis obliterans syndrome and control lungs, which had a similar number of airway bifurcations (Fig. 2a). Stratification of the graft vs. host disease airway counts according to obstructive and restrictive graft vs. host disease showed that this increase in the number of airways was exclusively seen in the restrictive graft vs. host disease group ( $p=0.014$ ) (Fig. 2b), whereas the obstructive graft vs. host disease lungs and the bronchiolitis obliterans syndrome lungs showed a similar number of airways. A higher proportion of obstruction was found in the obstructive graft vs. host disease compared with the restrictive graft vs. host disease group $(p<0.0001)$ (Fig. 2c). Indeed, within the obstructive graft vs. host disease group, airway obstruction was observed starting from fifth generation of branching. From the ninth generation on, $\sim 50 \%$ of the airways were obstructed and this increased up to $70-100 \%$ from generation 14. Interestingly, the proportion of airway obstruction in obstructive graft vs. host disease essentially mirrored that of bronchiolitis obliterans syndrome post lung transplantation (Fig. 2c). There was also a large similarity between restrictive graft vs. host disease and restrictive allograft syndrome, as significant airway obstruction (between 20\% and $35 \%$ of airways) was observed starting from generation 


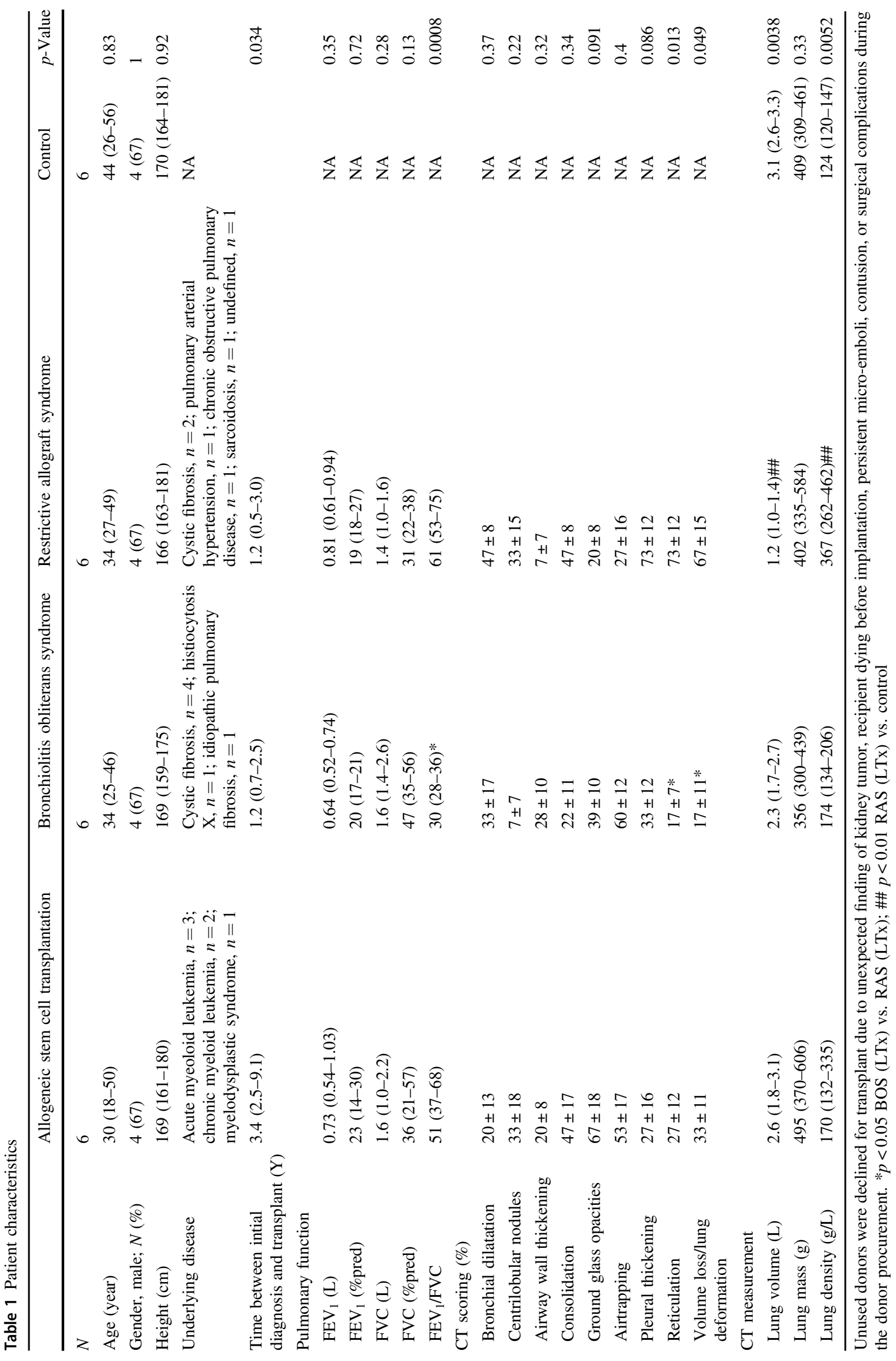


Table 2 Individual patient characteristics of the graft vs. host disease lungs

\begin{tabular}{|c|c|c|c|c|c|c|}
\hline & Obstructive1 & Obstructive2 & Obstructive3 & Restrictive1 & Restrictive2 & Restrictive3 \\
\hline Age at lung transplantation & 28 & 18 & 65 & 17 & 45 & 32 \\
\hline Height & 190 & 160 & 165 & 172 & 176 & 161 \\
\hline Weight & 80 & 43 & 59 & 51 & 51 & 66 \\
\hline Gender & Male & Male & Female & Male & Male & Female \\
\hline Native hematologic disease & $\begin{array}{l}\text { Acute myeoloid } \\
\text { leukemia }\end{array}$ & $\begin{array}{l}\text { Acute myeoloid } \\
\text { leukemia }\end{array}$ & $\begin{array}{l}\text { Chronic myeloid } \\
\text { leukemia }\end{array}$ & $\begin{array}{l}\text { Acute myeoloid } \\
\text { leukemia }\end{array}$ & $\begin{array}{l}\text { Chronic myeloid } \\
\text { leukemia }\end{array}$ & $\begin{array}{l}\text { Myelodysplastic } \\
\text { syndrome }\end{array}$ \\
\hline $\begin{array}{l}\text { Age at allogeneic stem cell } \\
\text { transplantation }\end{array}$ & 23 & 14 & 42 & 13 & 39 & 21 \\
\hline $\mathrm{FEV}_{1}(\mathrm{~L})$ & 1.18 & $0.98 *$ & 0.66 & 0.58 & 0.8 & 0.43 \\
\hline $\mathrm{FEV}_{1}(\%$ pred $)$ & 24 & $34 *$ & 28 & 14 & 21 & 14 \\
\hline FVC (L) & 3.23 & $1.9^{*}$ & 1.77 & 1.16 & 1.36 & 0.46 \\
\hline FVC (\%pred) & 55 & $43.3^{*}$ & 64 & 24 & 29 & 13 \\
\hline $\mathrm{FEV}_{1} / \mathrm{FVC}$ & 0.37 & $0.52 *$ & 0.37 & 0.50 & 0.59 & 0.93 \\
\hline \multicolumn{7}{|l|}{ CT scoring } \\
\hline Bronchial dilatation & 0 & 3 & 0 & 2 & 1 & 0 \\
\hline Centrilobular nodules & 1 & 1 & 1 & 0 & 3 & 0 \\
\hline Airway wall thickening & 0 & 2 & 1 & 1 & 1 & 0 \\
\hline Consolidation & 0 & 0 & 0 & 2 & 1 & 3 \\
\hline Ground glass opacities & 0 & 1 & 2 & 3 & 0 & 3 \\
\hline Airtrapping & 3 & NA & 1 & 2 & NA & 0 \\
\hline Pleural thickening & 1 & 0 & 0 & 1 & 0 & 2 \\
\hline Reticulation & 0 & 0 & 0 & 1 & 0 & 2 \\
\hline $\begin{array}{l}\text { Volume loss/lung } \\
\text { deformation }\end{array}$ & 0 & 0 & 0 & 2 & 1 & 1 \\
\hline
\end{tabular}

*This patient was not able to perform pulmonary function within 2 years of the lung transplant due to persistent pneumothorax. CT are scored from 0 (not present) -3 (highly present)

8 , increasing to $\sim 40 \%$ from generation 13 onwards. No obstructions were found in any of the control lungs.

\section{MicroCT imaging}

Using microCT (resolution of $10 \mu \mathrm{m}$ ) on tissue cores (96 tissue cores, 24 cores per group, 4 cores per lung), significant differences in the terminal bronchioles $/ \mathrm{ml}(p=0.042)$ and terminal bronchioles/lung $(p=0.0056)$ counts were found (Fig. 3). Indeed, control lungs showed a mean of 5.5 terminal bronchioles per $\mathrm{ml}$, which was significantly decreased in the allogenic hematopoietic stem cell transplantation group (2.9, $p<0.05$ ). The number of terminal bronchioles in the bronchiolitis obliterans syndrome and restrictive allograft syndrome group was not significantly different compared with control (4.5 and 3.9, respectively, $p<0.05$ ). Taking the total lung volume into account, a similar pattern was seen with a comparable number of terminal bronchioles in bronchiolitis obliterans syndrome and control, and a significant decrease in the allogenic hematopoietic stem cell transplantation group $(p<0.05)$, whereas the restrictive allograft syndrome group also had significantly lower numbers compared with control $(p<0.01)$. There was no difference in the diameter of the terminal bronchioles between the different groups $(p=0.68)$.

When comparing the obstructive graft vs. host disease and restrictive graft vs. host disease lungs, a similar pattern to lung transplantation was found: a twofold higher number of terminal bronchioles per lung in the obstructive graft vs. host disease group compared with the restrictive graft vs. host disease lung, although this was not statistically significant $(p=0.40)$. The surface density (an estimate of parenchymal fibrosis, with lower values representing more tissue fibrosis) was also different across the groups $(p=0.0059)$, although this difference could not be attributed to a specific difference between groups.

Interestingly, there was a significant correlation with surface density and the number of terminal bronchioles $/ \mathrm{ml}$ of tissue in the restrictive allograft syndrome group $(p=$ $0.02, R=0.47$ ), indicating that areas with more fibrosis have a lower number of terminal bronchioles compared with more normal appearing areas. 


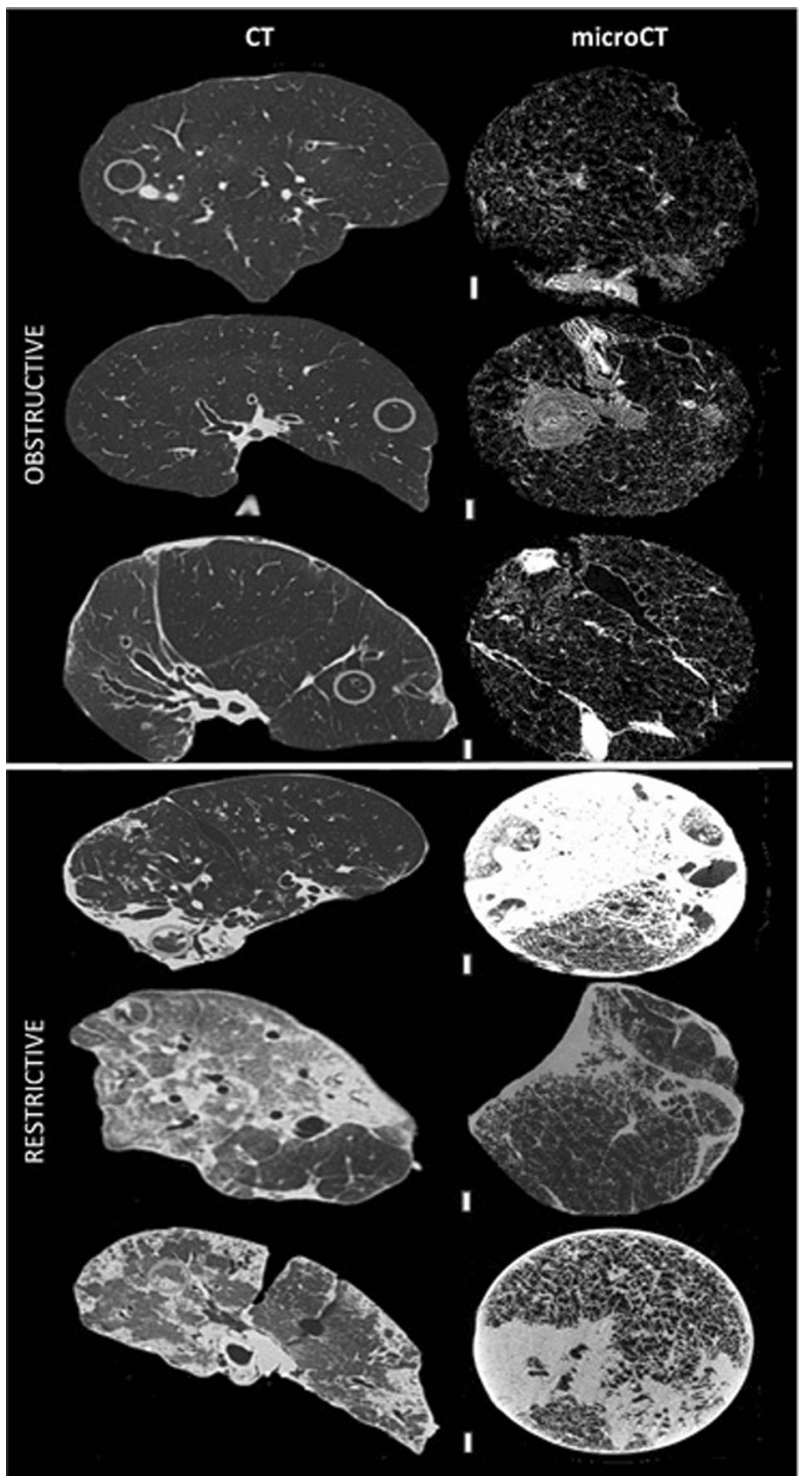

Fig. $1 \mathrm{CT}$ and microCT images of the six included allogenic hematopoietic stem cell transplantation lungs. The lungs presenting with an obstructive pulmonary function in general showed normal lung parenchyma with some airtrapping and centrilobular nodules (upper panel). The restrictive lungs showed severe ground glass opacification, consolidation, and volume loss (lower panel). The orange circles indicate the area where the sample for microCT examination was extracted. MicroCT confirmed a preservation of the normal lung parenchyma in the obstructive graft vs. host disease lungs, whereas areas of severe fibrosis and pleural thickening were observed in the restrictive graft vs. host disease lungs. The scale bars indicates $1 \mathrm{~mm}$

\section{Whole lung microCT imaging of graft vs. host disease lungs}

Although the resolution of ex vivo CT scanning $(700 \mu \mathrm{m})$ is improved compared with in vivo CT imaging, small airway obstruction can still be underestimated, especially as only small volume tissue cores were scanned. Therefore, we performed ex vivo whole lung microCT at a voxel size of
$120 \mu \mathrm{m}$ in one obstructive graft vs. host disease lung and one restrictive graft vs. host disease lung. Airway obstruction could be identified as illustrated in Fig. 4. The whole lung microCT shows an abundant presence of airway obstruction (green) across the airway tree (red, Fig. 5a). Indepth investigation shows that the airways are only obstructed for a certain portion of the airway, after which the airway re-opens as demonstrated in Fig. 5b. A total of 599 obstructions were detected at the ends of the airway branches (total 845, 70.9\%) within this lung. In the lower lobe, we detected a higher proportion of obstructed airways $(264 / 315,83.8 \%)$ compared with the upper/middle lob $(335 / 530,63.2 \%)$. In the whole lung microCT scan of the restrictive graft vs. host disease lung (Fig. 4c), central areas of fibrosis can be seen in yellow. Airway obstructions are also detected, although the relative proportion is lower compared with obstructive graft vs. host disease (169 obstruction $/ 428$ open airway ends $=39.5 \%$ ). Airway obstructions were often observed in areas of ongoing fibrosis as shown in Fig. 4d.

\section{Pathology}

Routine pathological examination showed that the obstructive graft vs. host disease lungs had evidence of bronchiolar obstruction, with limited, predominant lymphocytic, peribronchiolar inflammation. One lung also showed evidence of bronchiectasis, whereas in another Aspergillus nodules were found. The restrictive graft vs. host disease lungs showed interstitial fibrosis, with patchy interstitial inflammation. In one explant specimen, areas of honeycombing were found, whereas another also had evidence of emphysema, the subject being an ex-smoker with ten pack-years of smoking.

By further aligning serial microCT images of isolated obstructed airways with corresponding H\&E images of the same airways, we detected two different types of airway lesions, particularly in the obstructive graft vs. host disease lungs: the typical obliterative bronchiolitis lesion and a collapse lesion. Obliterative bronchiolitis typically shows a complete obstruction of the airway by extracellular matrix during which the airway preserves its shape and after which the airway re-opens (serial microCT and H\&E images through a single lesion in Fig. 6) at the level of the terminal bronchioles. Within the obliterative bronchiolitis lesions, a high number of vessel can be observed (Fig. 6o). The collapse lesion was characterized by an irreversible collapse of the airway in the absence of additional extracellular matrix accumulation (serial microCT and H\&E images trough a single lesion in Fig. 7). Without the sequential images provided by microCT, the lesion shown in Fig. 7h would hardly be recognizable as the remnant of an airway and would likely have remained undetected. 

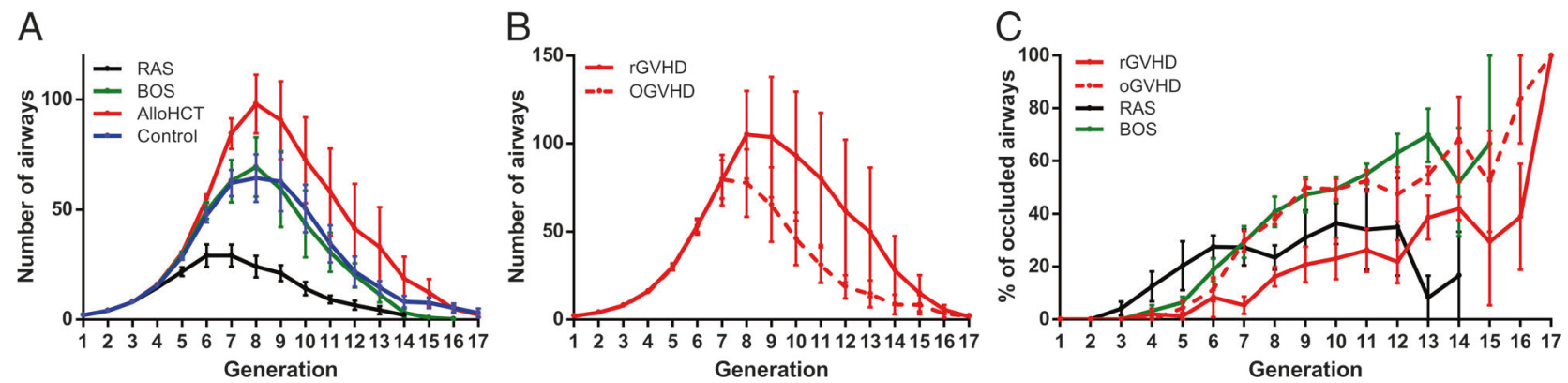

Fig. 2 CT airway and airway obstruction counts. a Comparison of the total number of airway bifurcations per airway generation across the study groups showing a higher number in allogenic hematopoietic stem cell transplantation and a lower number in restrictive allograft syndrome. b Airway counts in the graft vs. host disease group demonstrating that the number of airways in restrictive graft vs. host

disease are higher than obstructive graft vs. host disease lungs. c Degree of airway obstruction showing similar degree of obstruction in obstructive graft vs. host disease and bronchiolitis obliterans syndrome and restrictive graft vs. host disease and restrictive allograft syndrome. There is no airway obstruction in the control group
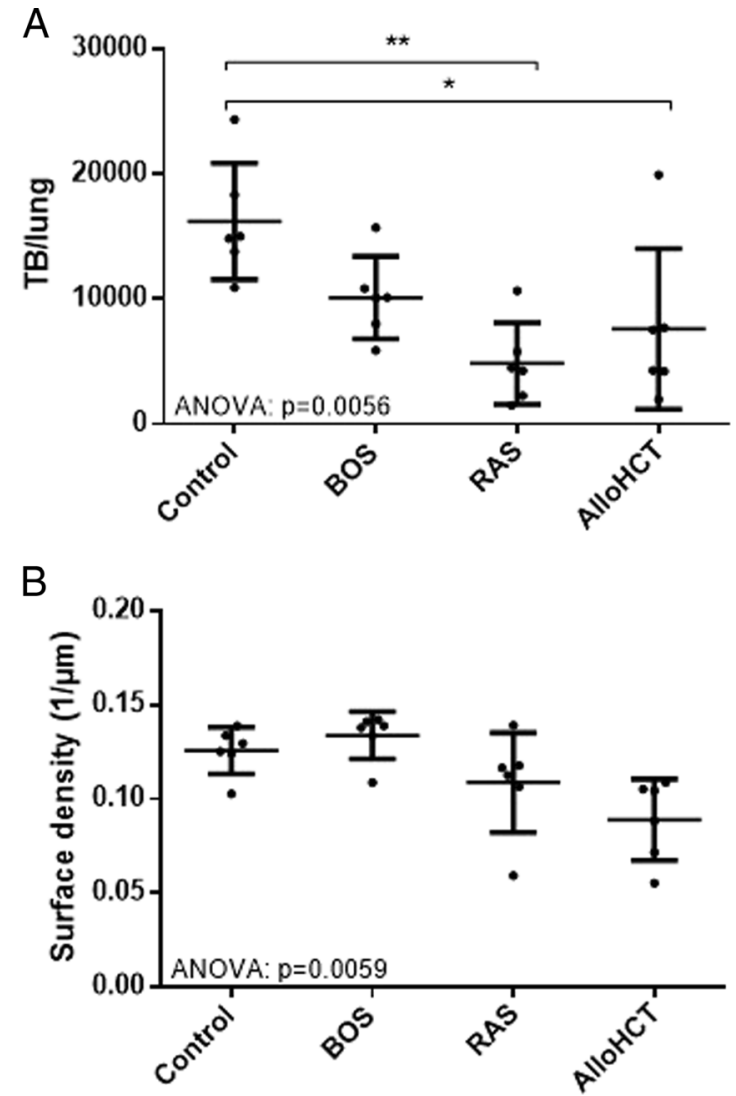

Fig. 3 Results of microCT analysis. a Scatterplot showing that the total number of terminal bronchioles per lung is lower in the allogenic hematopoietic stem cell transplantation and restrictive allograft syndrome group. b Surface density as a measurement of the size of the alveoli (lower surface density corresponds to more tissue fibrosis) show a lower number in the allogenic hematopoietic stem cell transplantation group and restrictive allograft syndrome group. ${ }^{*} p<0.05$ vs. control; $* * p<0.01$ compared with control

\section{Discussion}

Changes of airway and parenchymal morphometry in chronic graft vs. host disease after allogenic hematopoietic

stem cell transplantation was compared with phenotypes of chronic lung allograft dysfunction following lung transplantation and control lungs, using a multi-modal approach including CT-microCT and histology. A strong resemblance was found between bronchiolitis obliterans syndrome after lung transplantation and obstructive graft vs. host disease: a severe airway obstruction from generation 7 onward, with $70.8 \%$ of airways ending in obstruction and a more distally re-opening of the lumen as demonstrated on histology. Restrictive graft vs. host disease showed similarities to restrictive allograft syndrome post lung transplantation. It was characterized with a lower proportion of airway obstruction $(39.5 \%)$ and signs of injury to the lung parenchyma as evidenced by a lower number of terminal bronchioles and decrease in surface density. This suggests that the airways are similarly affected in posttransplant chronic lung allograft dysfunction and chronic graft vs. host disease post allogenic hematopoietic stem cell transplantation.

Based on this data, the hypothesis that obstructive graft vs. host disease after allogenic hematopoietic stem cell transplantation and bronchiolitis obliterans syndrome after lung transplantation are similar processes seems reasonable. In the graft vs. host disease setting, lung injury is not linked to previous ischemia reperfusion injury to the graft as seen in the lung transplantation setting. Therefore, it is likely that the similar presentation is rather due to a similar underlying immunological response with a plausible central role of the airway epithelium and T-cell-mediated injury [18]. This might also explain why similar therapeutic options seem to be effective in these conditions, with the most common ones being azithromycin, montelukast, and extracorporeal photophoresis [24].

The whole lung microCT scan for the first time allows the calculation of the exact number of airway obstructions in a typical lung with obstructive graft vs. host disease/ bronchiolitis obliterans syndrome. We found in total 599 

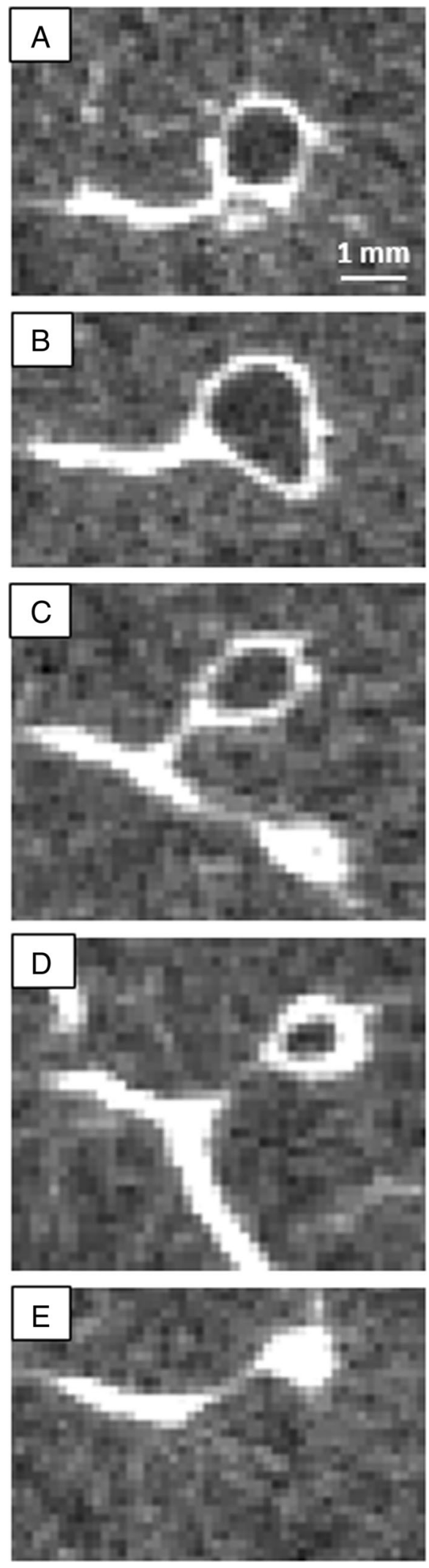

Fig. 4 Example of airway obstruction as observed on whole lung microCT imaging. An airway with a size of $1 \mathrm{~mm}$ is shown with the adjacent vessel (a). After bifurcating (b) the first daughter branch with a diameter of $800 \mu \mathrm{m}$ completely obliterates (c), in the following serial sections also the other daughter branch shows progressive obliteration of the lumen (d), leading to complete airway obstruction (e)

airway obstructions, which equals $70.8 \%$ of the total airways showing intermittent airway obstruction. These lesions were spread throughout the lung, but were more present in the lower lobe than the upper/middle lobe. This might be due to the lower ventilation/perfusion ratio in the lower lobes compared with the upper regions. We observed two types of airway obstruction, one that included additional matrix accumulation in the airway lumen and one where the airway seems to collapse, similar to the process we previously described in bronchiolitis obliterans syndrome [19]. This small airway disease can also be reliably detected using parametric response mapping, a CT-based technology where pixel to pixel comparison of inspiratory and expiratory allows the estimation of small airway disease and might be a future tool that can aid in the diagnosis of the disease [25] as its importance has been demonstrated in bronchiolitis obliterans syndrome following lung transplantation [26].

Within the patients undergoing lung transplantation for restrictive graft vs. host disease, a larger degree of heterogeneity in airway and parenchymal structures was evident. This is in line with literature as Schlemmer et al. [11], who identified significant heterogeneity on either CT (ground glass vs. consolidation) or histology (diffuse alveolar damage, organizing pneumonia, nonspecific interstitial pneumonia or lymphoid interstitial pneumonia) in an extended case series of 40 patients. Another pattern of interstitial lung disease following allogenic hematopoietic stem cell transplantation is pleuroparenchymal fibroelastosis [27]. The prevalence of pleuroparenchymal fibro-elastosis following allogenic hematopoietic stem cell transplantation is calculated to be $0.28 \%$ [28], which is much lower than the reported prevalence in restrictive allograft syndrome post lung transplantation (48-94\%) [16, 17]. Although we only included three patients with restrictive graft vs. host disease, the heterogeneity within this group is evident by the large SDs in the airway counts on CT and terminal bronchioles counted on microCT.

Recently, the study by Jonigk et al. [14] had performed a gene expression study to compare chronic lung graft vs. host disease and phenotypes of rejection using laser capture microdissection; obliterative bronchiolitis lesions following lung transplantation and allogenic hematopoietic stem cell transplantation were characterized by a similar gene expression signature of fibrosis-associated genes such as collagens, matrix metalloproteinases, and transforming 
Fig. 5 a Whole lung microCT imaging with a $120 \mu \mathrm{m}$ resolution showing the airway tree in an obstructive graft vs. host disease lung (airways in red), where airway obstructions are displayed in green, indicating a widespread abundance of small airway obstruction. b Demonstrates that the airway obliteration only obstructs a part of the airway, after which the airway re-opens and can sprout to normal terminal bronchioles. c Whole lung microCT imaging of a restrictive graft vs. host disease lung (airway in red), with areas of fibrosis highlighted in yellow and airway obstruction in green d An airway that disappears in an area of active fibrosis

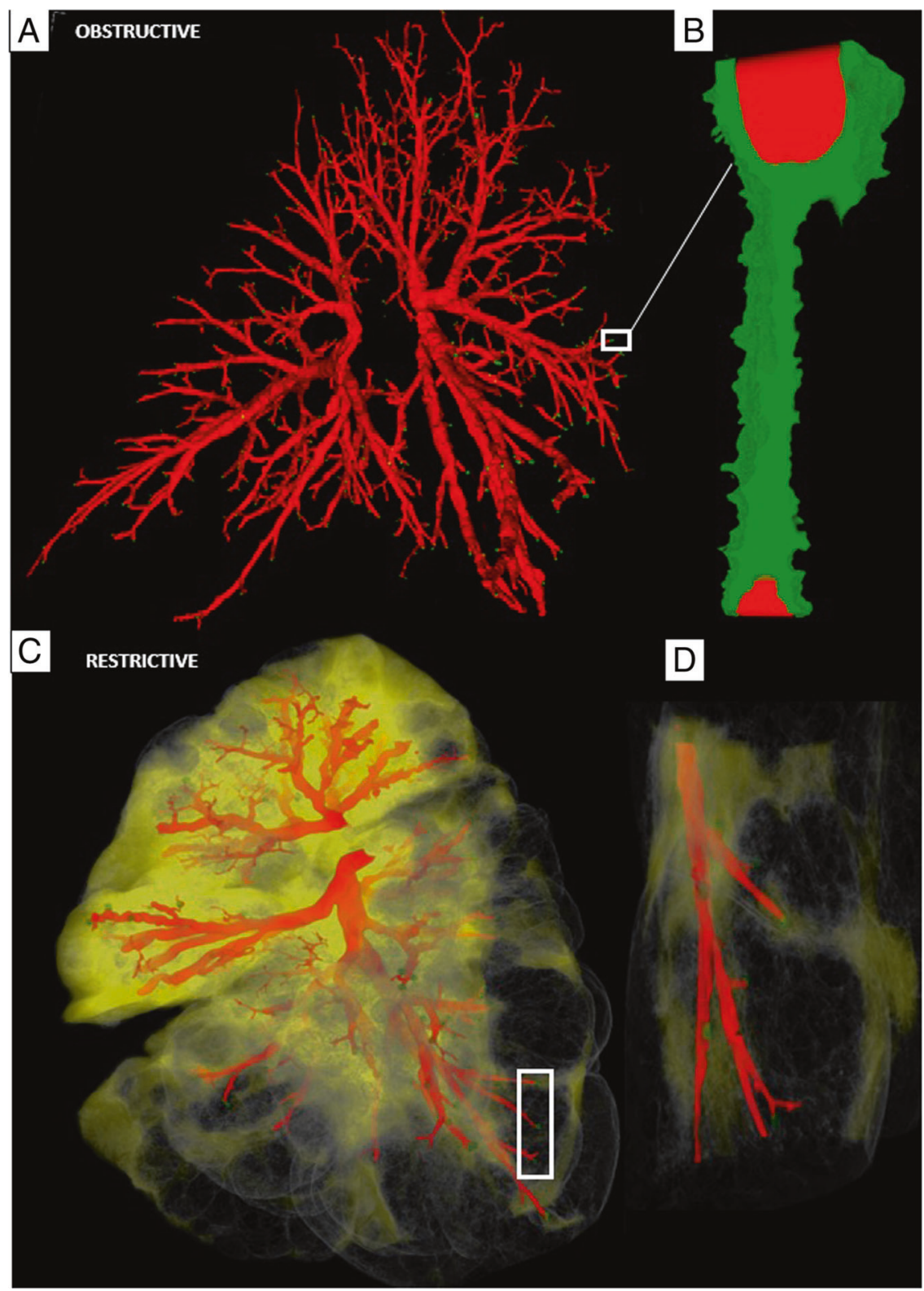

growth factor- $\beta$. Within the regions of alveolar fibrosis as observed in restrictive graft vs. host disease and restrictive allograft syndrome, some common patterns involved in remodeling of the extracellular matrix were also observed (collagen, matrix metalloproteinases), but the chronic graft vs. host disease lungs additionally showed fibrin upregulation, whereas the restrictive allograft syndrome lungs showed signs of fibroblast recruitment. Our airway morphometry analysis therefore largely confirm these similarities in molecular gene expression profile.

This study has several limitations such as the low number of graft vs. host disease lungs included in this study. Within allogenic hematopoietic stem cell transplantation, the number of graft vs. host disease lungs available for research is limited by the low incidence of lung graft vs. host disease, the difficulty to negotiate an autopsy with grieving families and the low number of chronic graft vs. host disease patients making it to lung transplantation. In fact, a recent study evaluating survival post lung transplantation in 20 European lung transplant centers over a 18-year period included 105 allogenic hematopoietic stem cell transplantation patients, the largest series described so far [29]. Therefore, the six patients included already represent a significant number. Ideally, the control lungs should all have been derived from allogenic hematopoietic stem cell transplantation recipients undergoing autopsy for non-pulmonary indications. However, autopsy is not systematically performed and often present confounding lung pathologies (infections, lung emboli, sepsis) making the interpretation challenging. The one included allogenic 
Fig. 6 MicroCT and H\&E of representative obliterative bronchiolitis lesion. An open airway $(\mathbf{a}-\mathbf{c})$ becomes completely obstructed (d-f, $\mathbf{g}-\mathbf{i}$, and $\mathbf{j}-\mathbf{l}$ ) and eventually opens up again $(\mathbf{m}-\mathbf{r})$ while maintaining a relative constant diameter. Panel I shows the inclusion of black particles in the lesion and in $\mathbf{o}$, a multitude of blood vessels are marked with * and also mononuclear infiltrates are discernible. Panel $\mathbf{r}$ demonstrates the constricting of the airway lumen by an increasing amount of tissue that is broadening the space between the smooth muscle layer (black arrow) and the epithelium (red arrow). Respective distance between the panels is based on serial microCT images $(\mathbf{a}-\mathbf{d}$ : $840 \mu \mathrm{m} ; \mathbf{d}-\mathbf{g}: 924 \mu \mathrm{m} ; \mathbf{g}-\mathbf{j}: 1260$ $\mu \mathrm{m}, \mathbf{j}-\mathbf{m}: 672 \mu \mathrm{m} ; \mathbf{m}-\mathbf{p}: 530 \mu \mathrm{m})$
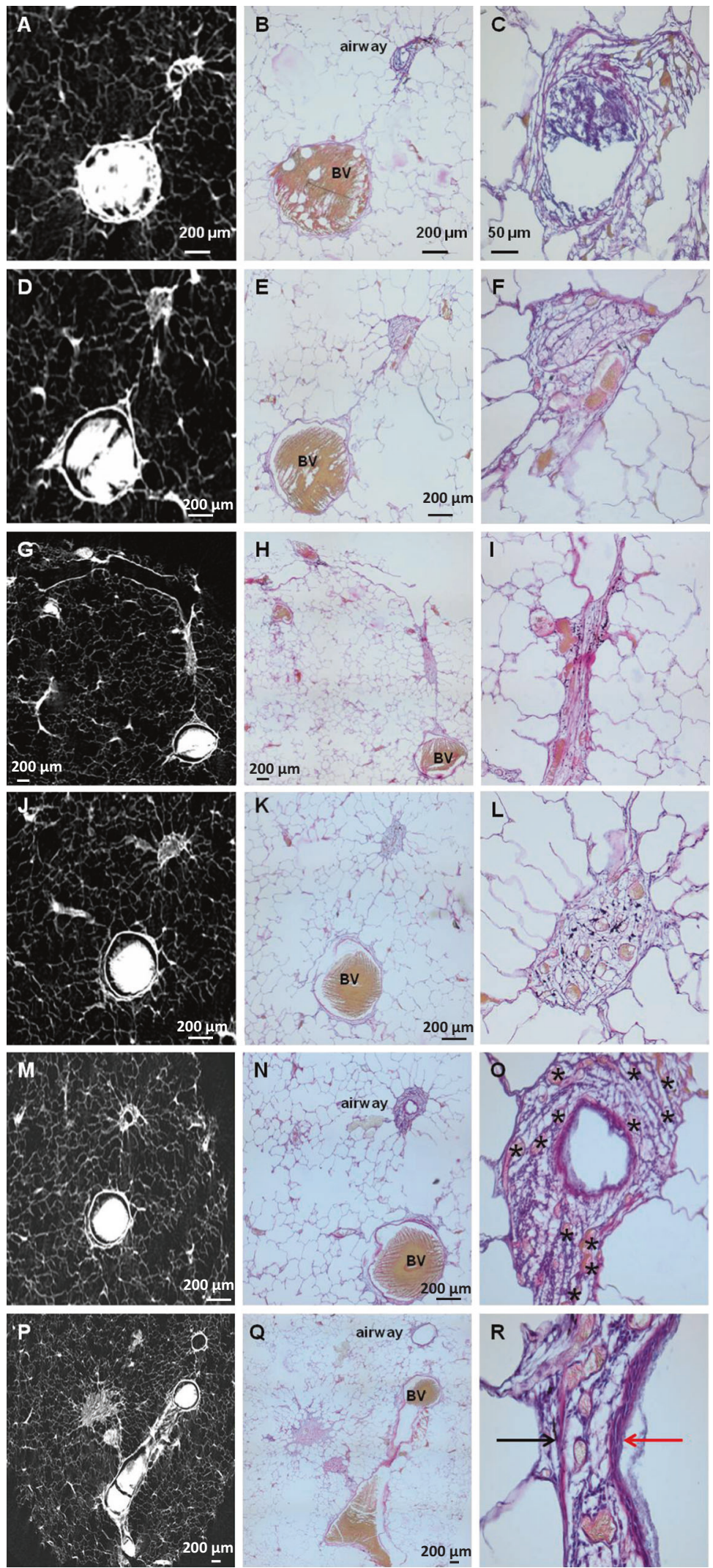
Fig. 7 MicroCT and H\&E of collapsed airway lesion. a An open airway with accompanying blood vessel on microCT is seen. b The corresponding histological image (panel b) shows some debris of unknown source in the airway lumen. $\mathbf{c}, \mathbf{d}$ shows a progressive subepithelial proliferation of fibroblasts leading to a complete obstruction (e,f), which eventually remodels to a scarlike lesion being the remnant of the airway (g,h). Respective distance between the panels is based on serial microCT images (a-c: $546 \mu \mathrm{m}$; c-e: $521 \mu \mathrm{m}$; e-g: $1462 \mu \mathrm{m})$
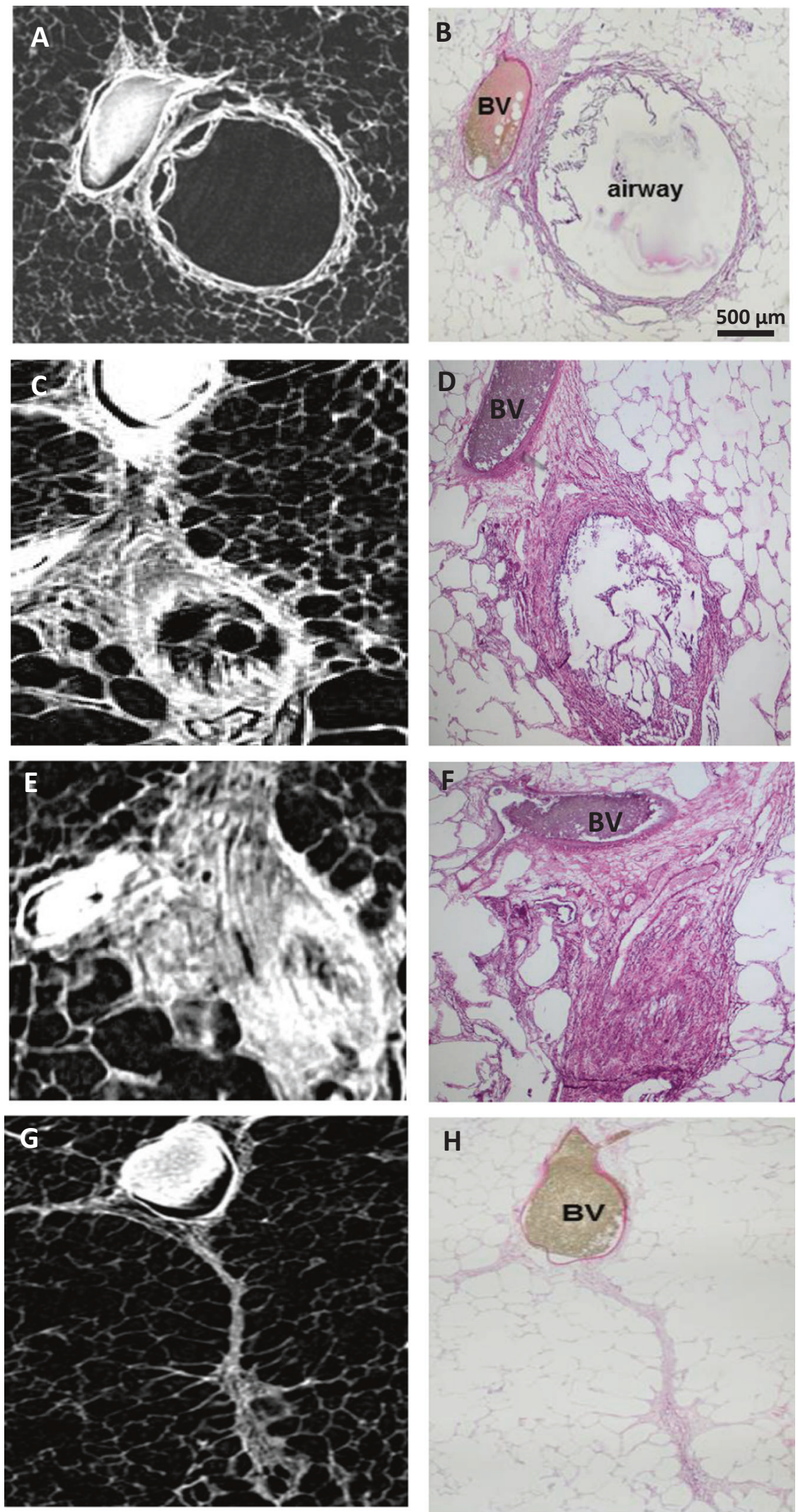
hematopoietic stem cell transplantation control without indications for pulmonary graft vs. host disease did not show evidence of airway obstruction and the other control lungs were well matched according to age and gender of the allogenic hematopoietic stem cell transplantation patients. Another limitation is the use of end-stage specimens, as the lungs in the end stage do not give insight in the early disease process. The freezing process applied, although necessary for microCT assessment, could complicate morphological interpretation, especially regarding airway obstruction. However, none of the control lungs showed evidence of airway obstruction, which provides further strength to our observed findings of airway obstruction and collapse.

In conclusion, we demonstrated the heterogeneity in the presentation of lung graft vs. host disease following allogenic hematopoietic stem cell transplantation. Moreover, there was a strong similarity in the morphometric changes within the lungs of patients presenting with obstructive and restrictive pulmonary function defects following allogenic hematopoietic stem cell transplantation and lung transplantation, suggesting similar underlying mechanisms that require further investigation.

Acknowledgements SEV is sponsored by a grant from the FWO (FWO12G8715N) and from KU Leuven (C24/18/073). BMV is funded by the KU Leuven (C24/050). RV is supported by a UZ Leuven starting grant (STG015/23) and FWO (senior clinical researcher). HS is supported by a $\mathrm{KOF} / \mathrm{KOOR}$ mandate of $\mathrm{UZ}$ Gasthuisberg. GV is supported by the Broere foundation. The Ghent University Special Research Fund (BOF-UGent) is acknowledged for the financial support to the Centre of Expertise UGCT (BOF.EXP.2017.000007).

\section{Compliance with ethical standards}

Conflict of interest The authors declare that they have no conflict of interest.

Publisher's note: Springer Nature remains neutral with regard to jurisdictional claims in published maps and institutional affiliations.

\section{References}

1. Gooley TA, Chien JW, Pergam SA, et al. Reduced mortality after allogeneic hematopoietic-cell transplantation. N Engl J Med. 2010;363:2091-101.

2. Hahn T, McCarthy PL, Hassebroek A, et al. Significant improvement in survival after allogeneic hematopoietic cell transplantation during a period of significantly increased use, older recipient age, and use of unrelated donors. J Clin Oncol. 2013;31:2437-49.

3. Solomon SR, Sizemore C, Zhang X, et al. Current graft-versushost disease-free, relapse-free survival: a dynamic endpoint to better define efficacy after allogenic transplant. Biol Blood Marrow Transplant. 2017;23:1208-14.

4. Solh MM, Bashey A, Solomon SR, et al. Long term survival among patients who are disease free at 1 -year post allogeneic hematopoietic cell transplantation: a single center analysis of 389 consecutive patients. Bone Marrow Transplant. 2018;53:576-83. https://doi.org/10.1038/s41409-017-0076-2.

5. Arai $\mathrm{S}$, Arora $\mathrm{M}$, Wang $\mathrm{T}$, et al. Increasing incidence of chronic graft-versus-host disease in allogeneic transplantation: a report from the Center for International Blood and Marrow Transplant Research. Biol Blood Marrow Transplant. 2015;21:266-74.

6. Abedin S, Yanik GA, Braun T, et al. Predictive value of bronchiolitis obliterans syndrome stage $0 \mathrm{p}$ in chronic graft-versushost disease of the lung. Biol Blood Marrow Transplant. 2015;21:1127-31.

7. Au BKC, Au MA, Chien JW. Bronchiolitis obliterans syndrome epidemiology after allogeneic hematopoietic cell transplantation. Biol Blood Marrow Transplant. 2011;17:1072-8.

8. Palmer J, Williams K, Inamoto Y, et al. Pulmonary symptoms measured by the national institutes of health lung score predict overall survival, nonrelapse mortality, and patient-reported outcomes in chronic graft-versus-host disease. Biol Blood Marrow Transplant. 2014;20:337-44.

9. Jagasia MH, Greinix HT, Arora M, et al. National Institutes of Health Consensus Development Project on Criteria for Clinical Trials in Chronic Graft-versus-Host Disease: I. The 2014 Diagnosis and Staging Working Group report. Biol Blood Marrow Transplant. 2015;21:389-401.e1.

10. Meignin V, Thivolet-Bejui F, Kambouchner M, et al. Lung histopathology of noninfectious pulmonary complications after allogeneic hematopoietic stem cell transplantation. Histopathology. 2018;73:832-42. https://doi.org/10.1111/his.13697.

11. Schlemmer F, Chevret S, Lorillon G, et al. Late-onset noninfectious interstitial lung disease after allogeneic hematopoietic stem cell transplantation. Respir Med. 2014;108:1525-33.

12. Sato M, Waddell TK, Wagnetz U, et al. Restrictive allograft syndrome (RAS): a novel form of chronic lung allograft dysfunction. J Heart Lung Transplant. 2011;30:735-42.

13. Chambers DC, Yusen RD, Cherikh WS, et al. The Registry of the International Society for Heart and Lung Transplantation: Thirty-fourth Adult Lung And Heart-Lung Transplantation Report-2017; focus theme: allograft ischemic time. J Heart Lung Transplant. 2017;36:1037-46. https://doi.org/10.1016/j. healun.2017.07.016.

14. Verleden SE, Ruttens D, Vandermeulen E, et al. Restrictive chronic lung allograft dysfunction: Where are we now? J Heart Lung Transplant. 2015;34:625-30.

15. Jonigk D, Rath B, Borchert $\mathrm{P}$, et al. Comparative analysis of morphological and molecular motifs in bronchiolitis obliterans and alveolar fibroelastosis after lung and stem cell transplantation. J Pathol Clin Res. 2017;3:17-28.

16. Ofek E, Sato M, Saito T, et al. Restrictive allograft syndrome post lung transplantation is characterized by pleuroparenchymal fibroelastosis. Mod Pathol. 2013;26:350-6.

17. von der Thüsen JH, Vandermeulen E, Vos R, et al. The histomorphological spectrum of restrictive chronic lung allograft dysfunction and implications for prognosis. Mod Pathol 2018;3:780-90. https://doi.org/10.1038/modpathol.2017.180.

18. Barker AF, Bergeron A, Rom WN, et al. Obliterative bronchiolitis. N Engl J Med. 2014;370:1820-8.

19. Verleden SE, Vasilescu DM, Willems S, et al. The site and nature of airway obstruction after lung transplantation. Am J Respir Crit Care Med. 2014;189:292-300.

20. Verleden SE, Vasilescu DM, McDonough JE, et al. Linking clinical phenotypes of chronic lung allograft dysfunction to changes in lung structure. Eur Respir J. 2015;46:1430-9.

21. Verleden SE, Ruttens D, Vandermeulen E, et al. Predictors of survival in restrictive chronic lung allograft dysfunction after lung transplantation. J Heart Lung Transplant. 2016;35: 1078-84. 
22. Hansell DM, Bankier AA, MacMahon H, et al. Fleischner Society: glossary of terms for thoracic imaging. Radiology. 2008;246:697-722.

23. Masschaele B, Dierick M, Loo DV, et al. HECTOR: a $240 \mathrm{kV}$ micro-CT setup optimized for research. J Phys: Conf Ser. 2013;463:12012.

24. Bergeron A, Cheng G-S. Bronchiolitis obliterans syndrome and other late pulmonary complications after allogeneic hematopoietic stem cell transplantation. Clin Chest Med. 2017;38:607-21.

25. Galbán CJ, Boes JL, Bule M, et al. Parametric response mapping as an indicator of bronchiolitis obliterans syndrome after hematopoietic stem cell transplantation. Biol Blood Marrow Transplant. 2014;20:1592-8.
26. Verleden SE, Vos R, Vandermeulen E, et al. Parametric response mapping of bronchiolitis obliterans syndrome progression after lung transplantation. Am J Transplant. 2016;16:3262-9.

27. Reddy TL, Tominaga M, Hansell DM, et al. Pleuroparenchymal fibroelastosis: a spectrum of histopathological and imaging phenotypes. Eur Respir J. 2012;40:377-85.

28. Mariani F, Gatti B, Rocca A, et al. Pleuroparenchymal fibroelastosis: the prevalence of secondary forms in hematopoietic stem cell and lung transplantation recipients. Diagn Interv Radiol. 2016;22:400-6.

29. Greer M, Berastegui C, Jaksch P, et al. Lung transplantation after allogeneic stem cell transplantation: a pan-European experience. Eur Respir J. 2018;51:pii: 1701330. 